\title{
Denoising electromyogram and electroencephalogram signals using improved complete ensemble empirical mode decomposition with adaptive noise
}

\author{
S. Elouaham ${ }^{1}$, A. Dliou ${ }^{2}$, N. Elkamoun ${ }^{3}$, R. Latif ${ }^{4}$, S. Said ${ }^{5}$, H. Zougagh ${ }^{6}$, K. Khadiri ${ }^{7}$ \\ ${ }^{1,3,7}$ LSTIC, Faculty of Sciences, Chouaib Doukkali University, El Jadida, Morocco. \\ ${ }^{1,2,4}$ LISTI, National School of Applied Sciences, Ibn Zohr University, Agadir, Morocco \\ ${ }^{5}$ Department of Physics, University of Mohamed Premier, Oujda, Morocco \\ ${ }^{6}$ Faculty of sciences and techniques, Sultan Moulay Slimane, University Beni Mellal, Morocco
}

\begin{abstract}
Article Info
Article history:

Received Apr 18, 2021

Revised Jul 6, 2021

Accepted Jul 14, 2021

Keywords:

CEEMDAN

EEG

EMG

ICEEMDAN

MAUPS

ABSTRACT

The health of the brain and muscles depends on the proper analysis of electroencephalogram and electromyogram signals without noise. The latter blends into the recording of biomedical signals for external or internal reasons of the human body. Therefore, to obtain a more accurate signal, it is needed to select filtering techniques that minimize the noise. In this study, the techniques used are empirical mode decomposition and its variants. Among the new versions of variants is the improved complete ensemble empirical mode decomposition with adaptive noise. These methods are applied to electroencephalogram and electromyogram signals corrupted by natural noise and white Gaussian noise. The obtained results through the use of the improved complete ensemble empirical mode decomposition with adaptive noises how the high performance that includes minimizing the noise and the effectiveness of the components of the signals used in the present research. This method has low values of the mean square error and high values of signal-to-noise ratio compared to other methods used in this study.
\end{abstract}

This is an open access article under the CC BY-SA license.

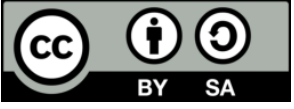

\section{Corresponding Author:}

Elouaham Samir

LSTIC, Faculty of sciences

Chouaib Doukkali University, El Jadida

Email: elouahamsamir@gmail.com

\section{INTRODUCTION}

Electroencephalogram (EEG) and electromyogram (EMG) signals are clinical tests that successively evaluate the work of the brain, muscles, and nerves. These measures are safe and painless. Such activities have been obtained by placing the electrode in a good position of the patient's muscles and scalp. The EEG signal contains good information about electrical movements [1], [2]. This latter is engendered by the cerebral cortex. The EEG signal can give the best assessment of brain activities and detect signal irregularities. The measures and determinations of the number of the motor unit action potential (MUAP) presented in the EMG signal, which gives beneficial information, are provided an exact idea of the work of muscles. MUAP is the wave that contains information about muscular dystrophy and neuropathies, nerve conduction, and neuropathies [3]-[5]. The electromyogram and electroencephalogram signals are non-linear and non-stationary [6], [7]. Due to the latter, denoising and analyzing these signals are very important. The Research on EEG and EMG signal filtering has been consistently a useful research area. Traditionally, correction of baseline wander (BW) is performed by non-linear denoising, which can simply result in a misrepresentation of the waveform [8]. Electrode motion (EM) noise is one of the most disturbing, because it is not always easily removed by conventional filters [8]. 
The muscle artifact (MA) noise is caused by muscle contraction, which coincides with the measurement of muscular activity; the amplitude of this noise may completely cover the biomedical signals [8]. The ocular artifacts (OA); these noises are the sources of eye movement and blinking. The changes in the direction of the cornea dipole, retina, and flutter produce the ocular noise [9].

The denoising techniques are inevitable for a reasonable interpretation of biomedical signals and the reduction of the noises that interfere with the EEG and EMG signal. Among the denoising approaches, empirical mode decomposition (EMD) and its variants. The EMD is a time-frequency information processing technique [10], [11]. The EMD information processing technique decomposes EMG and EEG signals into signals named intrinsic mode functions (IMFs), that have a high frequency for the first IMF and low frequency for the residual (the last IMF). The denoised EEG and EMG signals can be restored by the IMFs that have a low frequency and the residual signal (the high frequency existing in the first IMFs have noises). This denoising method is applied in several fields as a global positioning system, biomedical signals, and climate change [10]-[14]. The drawbacks of the EMD are the end effect and modal-aliasing. To overcome the problems created in the EMD tool, the white Gaussian noise is mixed with signaland the EMD is applied to the final signal results. The mean of the results obtained is ensemble EMD (EEMD) [15].

Despite the white noise added in practice to overcome mode-mixing by the EEMD technique, the noise in the signal exists after calculate the multiple averaging. The EEMD method also has constraints; for instance, the number of scales is not the same as the EMD decomposition, and the quantity of calculations increases [16]. The pitfalls that appeared in EMD and EEMD were overcome by applying complete ensemble empirical mode decomposition with adaptive noise (CEEMDAN) that uses the noise auxiliary. This method gives a correct restoration of biomedical signals and perfect spectra of the decomposed mode [17]-[19]. The iterations presented in EEMD are more than iterations of the CEEMDAN method. This latter may give a good reconstruction of the signals and reclaim the EMD characteristics that do not appear in EEMD. The residual noise and spurious modes are presented in the CEEMDAN method despite the amelioration of the EMD and EEMD. To surpass these problems created by CEEMDAN, the new technique called Improved CEEMDAN is used in this study to attain the modes with the filtered noise and give the signal a more physical meaning [20]-[24].

This article is structured as follows. Section 2 provides an explanation of the spectrum restoration technique based on the CEEMDAN and its new version. The signals used in this paper are also presented. Section 3 presents the results obtained through the use ofthese techniques. The final part sums up the main findings and conclusions of the study.

\section{DENOISING AND BIOMEDICAL SIGNALS}

\subsection{Denoising techniques}

\subsubsection{CEEMDAN}

The EMD is a method that decomposes the $\mathrm{z}(\mathrm{t})$ signal into modes called intrinsic mode function IMFs. The EMD method is presented by the following steps [6], [7]: the first step is the extraction of all extrema from the original signal and the second step is the calculation of the local average $m(t)$ :

$$
m(t)=\frac{\left(e_{\max }(t)+e_{\min }(t)\right.}{2}
$$

With $e_{\max }(t)$ is the upper envelope and $\left.e_{\min }(t)\right)$ is lower envelope. Then, the third step is computing the mode $\mathrm{d}_{\mathrm{J}}(\mathrm{t})=\mathrm{IMF}_{\mathrm{J}}(\mathrm{t})$, a local detail by:

$$
d(t)=z(t)-m(t)
$$

The fourth step is to compute the new signal $r(t)=z(t)-i m f_{1}(t)$, and to determine if $r(t)$ less than three extrema, the algorithm stops has; otherwise, pass to the first step.

The CEEMDAN technique is based on EMD. The set of rules and basics ofthe EMD theory and its variant techniques are given in [17]-[19]. The CEEMDAN method is presented as follows:

- Decompose $x^{i}(n)+w_{0} \varepsilon^{i}(n)$ to access the first mode by (3):

$$
\overline{I M F}_{1}(n)=\frac{1}{I} \sum_{i=1}^{I} \overline{I M F_{k}^{i}}(n)
$$


where $w_{0}$ is the level noise adding in signal, $\varepsilon^{i}(t)$ is a different white Gaussien noise $(\mathrm{i}=1, \ldots, \mathrm{I})$ and $\mathrm{k}$ represents the mode.

- $\quad$ The first residue is obtained by the following relation:

$$
r_{1}(n)=x(n)-\overline{I M F}_{1}(n)
$$

The Operator Ej(.) presents the ith mode obtained by decomposition of the biomedical signals (EEG or EMG) with the EMD method.

- The new signal $r_{1}(n)+w_{1} E_{1}\left(\varepsilon^{i}(n)\right)$ is decomposed to obtain the first mode. The $\overline{I M F}$ is given by (5):

$$
\overline{I M F}_{2}(n)=\frac{1}{I} \sum_{i=1}^{I} E_{1}\left(r_{1}(n)+w_{1} E_{1}\left(\varepsilon^{i}(n)\right)\right)
$$

For $\mathrm{k}=2, \ldots, \mathrm{K}$, calculate the $\mathrm{k}$-th residue and obtain the first mode. Define the $(\mathrm{k}+1)$-th mode as $(6)$ :

$$
\overline{I M F}_{k+1}(n)=\frac{1}{I} \sum_{i=1}^{l} E_{1}\left(r_{k}(n)+w_{k} E_{k}\left(\varepsilon^{i}(n)\right)\right)
$$

- $\quad$ Iterate this operation until the residual is no longer feasible. The residue is obtained by (7).

$$
r_{k}(n)=r_{k-1}(n)-\sum_{k=1}^{K} \overline{I M F}_{k}
$$

The final equation of residue is obtained as (8):

$$
r(n)=x(n)-\sum_{k=1}^{K} \overline{I M F}_{k}
$$

After decomposition of the EEG, EMG signal, the results of the final signal is obtained by (9):

$$
x(n)=r(n)+\sum_{k=1}^{k} \overline{I M F}_{k}
$$

\subsubsection{Improved CEEMDAN (ICEEMDAN)}

Improved complete ensemble empirical mode decomposition with adaptive noise (ICEEMDAN) method is based on the last technique cited above, named CEEMDAN method [23]-[25]: The operator M(.) computes the local mean of the EEG or EMG signal in this research. The ICEEMDAN method is obtained by the following steps. The signal $x^{i}(n)=x(n)+\beta_{0} E_{1}\left(w^{(i)}(n)\right)$ is original with white Gaussien noise where: $\beta_{0}=\frac{\varepsilon_{0} \operatorname{std}(x)}{\operatorname{std}\left(E_{1}\left(w^{i}(n)\right)\right)}$

The white Gaussian noise is important to calculate and generate the new extrema with the new version of the EMD method. These new extrema are more important to give the new local mean. The local means is calculated using realization I of the EMD technique applied on the biomedical signal to obtain the first residue: $r_{1}=\left\langle M\left(w^{(i)}\right)\right\rangle$

- $\quad$ The mode for $\mathrm{k}=1$ is:

$$
\overline{I M F}_{1}=x(n)-r_{1}
$$

The next residue presented by this equation: $r_{1}+\beta_{1} E_{2}\left(w^{(i)}(n)\right)$; then the $\overline{I M F}_{2}$ is obtained by (11):

$$
\overline{I M F}_{2}=r_{1}-r_{2}=r_{1}-\left\langle M\left(r_{1}+\beta_{1} E_{2}\left(w^{(i)}(n)\right)\right)\right\rangle
$$

- $\quad$ For $\mathrm{p}=3, \ldots, \mathrm{P}$ calculate the pth residue $r_{p}=\left\langle M\left(r_{p-1}+\beta_{p-1} E_{p}\left(w^{(i)}(n)\right)\right)\right\rangle$

- $\quad$ Calculates the pth mode

$$
\overline{I M F}_{p}=r_{p-1}-r_{p}
$$




\subsection{Biomedical informations}

The EEG and EMG signals are used in this paper, these signals are naturels and provides by the MIT-BIH database [8]. Testing of these signals is non-invasive; they can show the normalities and abnormalities of brain and muscle functions in humans. The abnormal signal presented in the EEG signal can be represented by the sleep disorder in the case of this research. The first subject presented in this paper is healthy for EEG signal and the secondone is abnormal. Figure 1 shows the EEG signal in case of neurological disorders and Figure 2 shows the abnormal EEG signal

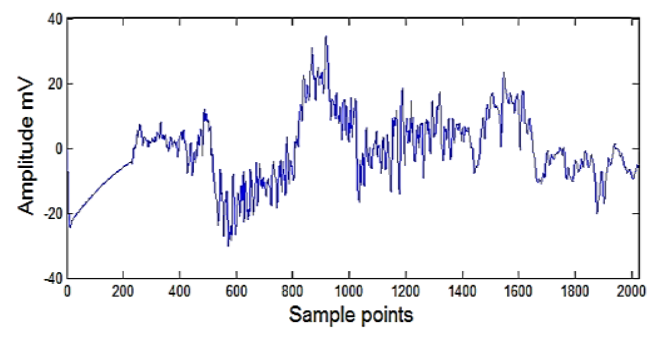

Figure 1. Normal EEG signal

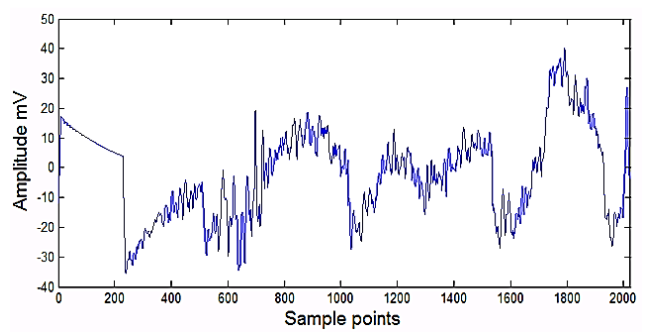

Figure 2. Abnormal EEG signal

The electromyogram test can give more information about the states of the human muscles. EMG signals have a good factor named MUAPs which gives the morphology, amplitude, distance between two successive MUAPs, and the frequency content of this last. Figure 3 shows the EMG signal with neuropathy and Figure 4 presents the EMG signal with myopathy.

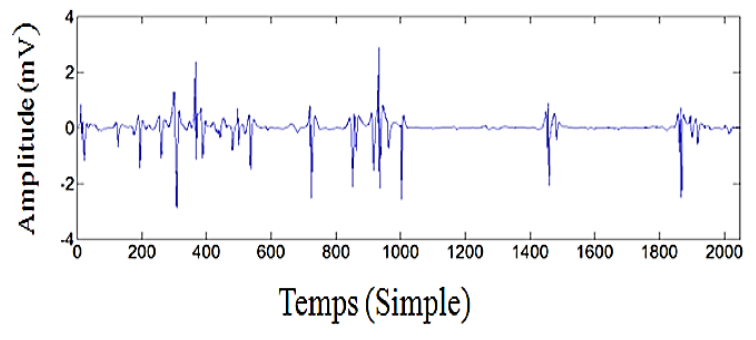

Figure 3. Abnormal EMG signal: Neuropathy

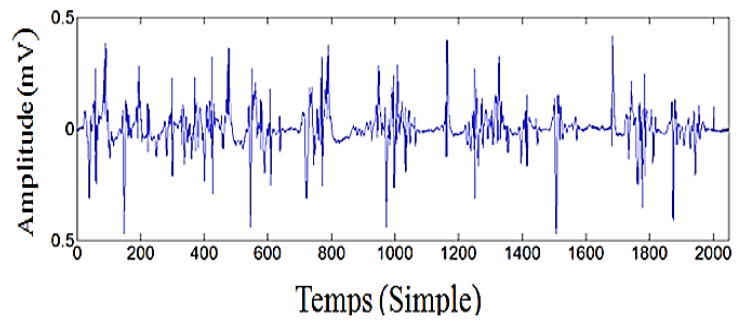

Figure 4. Abnormal EMG signal: Myopathy

The noises signals used in this research are muscle artifacts (MA), Basline wander (BW) and electrod motion (EM) [8]. This first noise shown in Figure 5 is a muscle artifact. This noise is a familiar high frequency signal and is a natural noise provided by the MIT-BIH Database[8]. The spectrum of the muscle artifact interferes with the biomedical signals.

This noise shown in Figure 6 is the baseline wander. This noise is the origin of the patient's movement. or the leads. The baseline wander is a low-frequency family and is a natural noise provided by the MIT-BIH database [8]. The electrode motion noise is one of the difficult noises to minimize because it can imitate the ECG signal components. Generally is obtained by intermittent mechanics. This artifact cannot be deleted without difficulty by conventional methods, as can other noise and is a natural noise provided by the MIT-BIH database [8]. Figure 7 shows the electrod motion (EM) artifact.

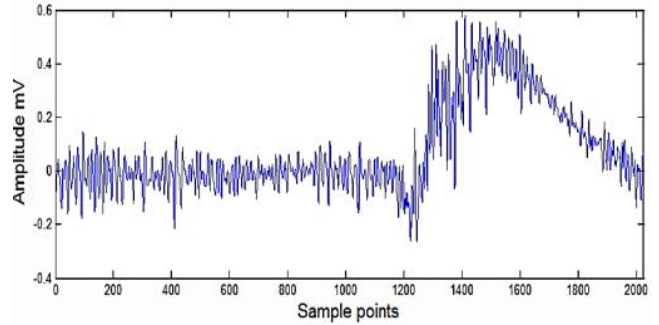

Figure 5. Noise signal muscle artefact (MA)

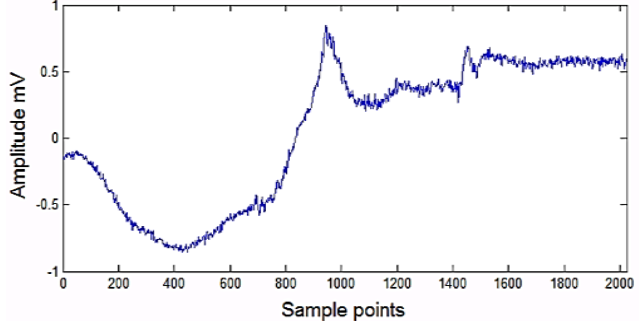

Figure 6. Noise signal Baseline wander (BW) 


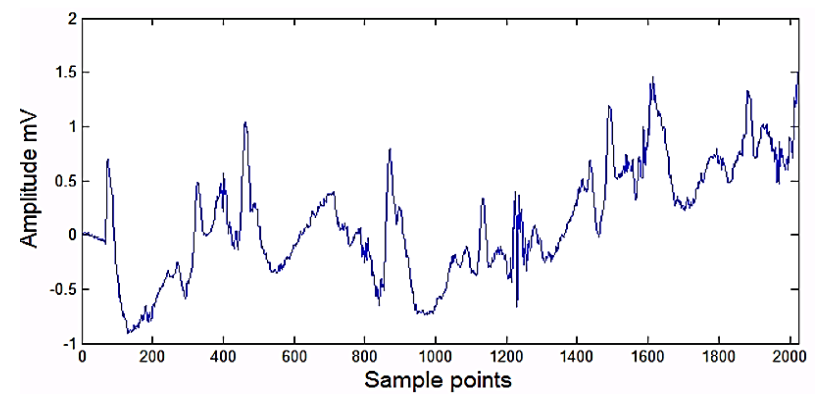

Figure 7. Noise signal electrod motion (EM)

The creation of the noise as white noise, BW, MA, and EM needs functions in MATLAB that permit it to generate. In this research, we used a function that associated the natural noises as BW, MA, and EM, this function is $\mathrm{CN}$. The $\mathrm{CN}$ indicates the realistic artifact that impedes the EEG and EMG registration. The CN noise interfered with the real and clean EEG and EMG signals, and the white noise is added with different values of the signal-to-noise ratio (SNR). CN composed noise is given by following equation [17]:

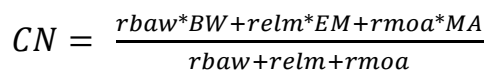

The percentage of noise added to the BW is defined by rbaw $=2$, theEM noise is defined by relm $=2$, and the MA noises are defined by rmoa=5. The evaluation of the results obtained by the algorithms used in this research using the indicators the SNR improvement $S N R_{O U T}$ and MSE. The low MSE values and high SNR_imp values indicate the effect performs of the denoising methods. The metrics are calculated as (14):

$$
M S E=\frac{1}{k} \sum_{k=1}^{k}(z(n)-\bar{z}(n))^{2}
$$

The SNR equation indicates the robustness of the methods by giving high values that demonstrate the performance of denoising and restoration of signal shapes, on the other hand, the low values of MSE shows the powerful method. The $S N R_{\text {OUT }}$ equation is obtained by (15):

$$
S N R_{\text {OUT }}(d B)=10^{*} \log _{10}\left(\frac{\sum_{i=0}^{K-1}[z(n)]^{2}}{\sum_{i=0}^{K-1}[z(n)-\bar{z}(n)]^{2}}\right)
$$

with $\mathrm{z}(\mathrm{n})$ is the EEG signal before adding the noise, $\bar{z}(n)$ is results after de-noising the EEG and EMG signals and $\mathrm{N}$ is the number of biomedical length signals used.

\section{RESULTS AND DISCUSSION}

The EMG signals generally interfere with natural noises that hide useful information. This information has more significant meaning that explains how the muscles work and gives where the problems exist. In this research, EMG signals are contaminated by the composed noises; containing EM, BW, MA and white noise. We used (13); this equation combines the four noises cited above. The denoising techniques applied on the EMG signals are the CEEMDAN method and its ICEEMDAN variant. The filtering techniques are applied on abnormal myopathy and neuropathy signals with different SNR. Table 1 shows the results obtained by these methods. Figure 8 presents the MSE of abnormal EMG signal with neuropathy.

Table 1. MSE of normal and abnormal EMG signals

\begin{tabular}{ccccc}
\hline Signal SNR “dB" & \multicolumn{2}{c}{ Myopathy } & \multicolumn{2}{c}{ Neuopathy } \\
& ICEEMDAN & CEEMDAN & ICEEMDAN & CEEMDAN \\
\hline 0 & 0.01075 & 0.01077 & 0.23049 & 0.23102 \\
5 & 0.00439 & 0.00440 & 0.10144 & 0.10194 \\
10 & 0.00236 & 0.00237 & 0.06064 & 0.06107 \\
15 & 0.00171 & 0.00171 & 0.04803 & 0.04826 \\
\hline
\end{tabular}




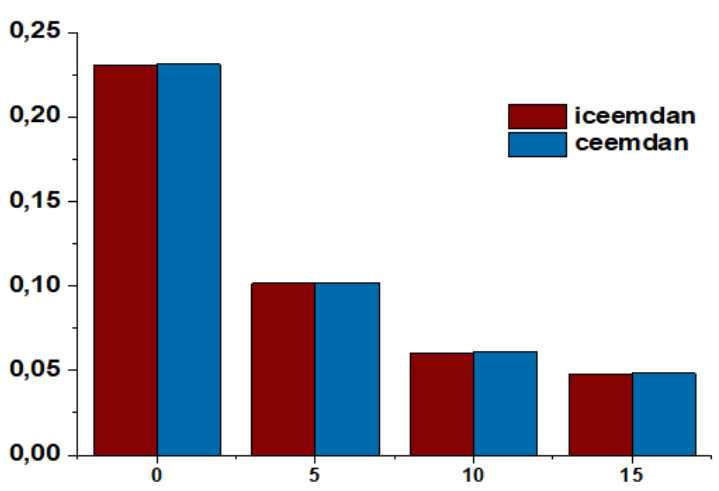

Figure 8. EMG signal with neuropathy

The EEG signals can be contaminated by ocular artifacts (OA); these noises are the sources of eye movement and blinking. The changes in the direction of the cornea dipole, retina and flutter produce the ocular noise. EEG and EOG can propagate to the surface of the scalp, through the medium of volume conduction effect. Table 2 presents the results of the SNR to the abnormal EEG signal. Table 3 presents the results of the SNR compared to normal EEG signal. Figures 9 and 10 present the SNR evaluation of normal and abnormal EEG signals contaminated by EOG signal and white noise with $10(\mathrm{~dB})$ et $20(\mathrm{~dB})$. The choice of the $10 \mathrm{~dB}$ and $20 \mathrm{~dB}$ is related to the level of noise that can be in the middle between the high and low values of the SNR. The metric used for evaluation and comparison the de four denoising methods are SNR given by (13).

The Tables 2 and 3 show the robustness of the new technique called ICEEMDAN that presents the high values $(19,9330472,10,0872857)$ of SNR $20 \mathrm{~dB}$ and 10dB for the normal EEG signal compared with the CEEMDAN (19,8867641;10,0862754) EEMD (19,7109528; 10,0421496) EMD (17,6338571;9,7408917) and the same results are obtained for the abnormal EEG signal. The Table 1 presents the low values of the MSE obtained by the denoising method called ICEEMDAN compared with the CEEMDAN technique. The MSE of the abnormal EMG signal with myopathy and neuropathy obtained by ICEEMDAN $(0,01075$; 0,23049) CEEMDAN (0,01077; 0,23102). The quality of the signal is depending on the level of the restoration and the elimination of the noise that interfered with the biomedical signal used in this paper. From the results, the ICEEMDAN method shows high performance and power according to the results obtained from the MSE and SNR metrics; when reforming the EEG and EMG signals with composite noise filtering, the results obtained are better than those obtained in our research published in these results [7].

Table 2. $S N R_{O U T}$ of denoising techniques

\begin{tabular}{lcc}
\hline \multicolumn{1}{c}{ SNR en dB } & $10 \mathrm{~dB}$ & $20 \mathrm{~dB}$ \\
\hline ICEEMDAN & 10.0948868 & 20.0256902 \\
CEEMDAN & 10.0883071 & 19.9886309 \\
EEMD & 10.0456369 & 19.5118041 \\
EMD & 10.0091271 & 18.9140325 \\
\hline
\end{tabular}
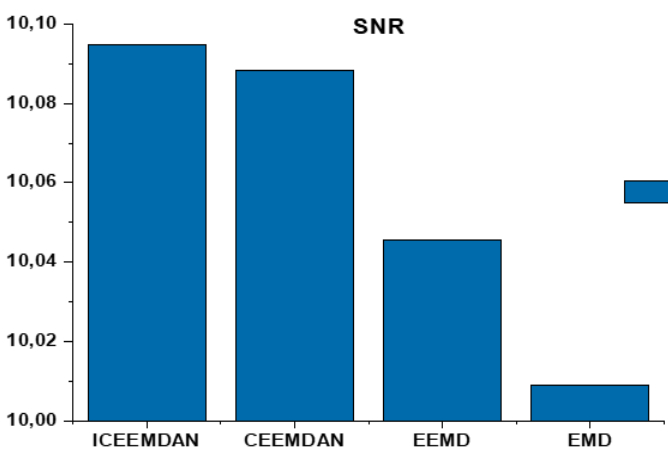

(a)
Table 3. $S N R_{O U T}$ of denoising techniques

\begin{tabular}{lcc}
\hline \multicolumn{1}{c}{ SNR en dB } & $10 \mathrm{~dB}$ & $20 \mathrm{~dB}$ \\
\hline ICEEMDAN & 10.0872857 & 19.9330472 \\
CEEMDAN & 10.0862754 & 19.8867641 \\
EEMD & 10.0421496 & 19.7109528 \\
EMD & 9.7408917 & 17.6338571 \\
\hline
\end{tabular}

$10 \mathrm{~dB}$

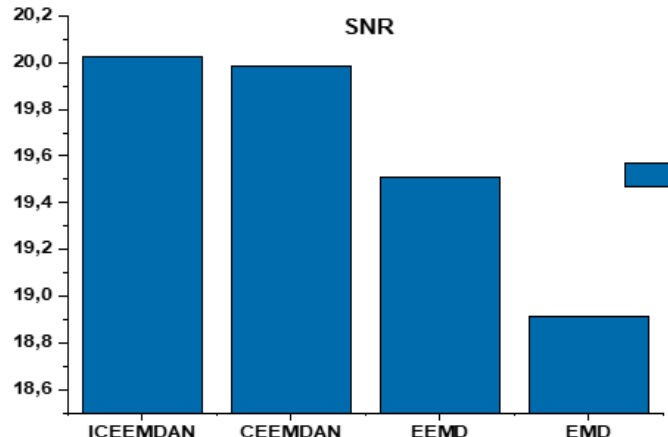

(b)

Figure 9. Diagram of SNR evaluation for abnormal EEG signal denoising for; (a) $10 \mathrm{~dB}$ and (b) $20 \mathrm{~dB}$ 


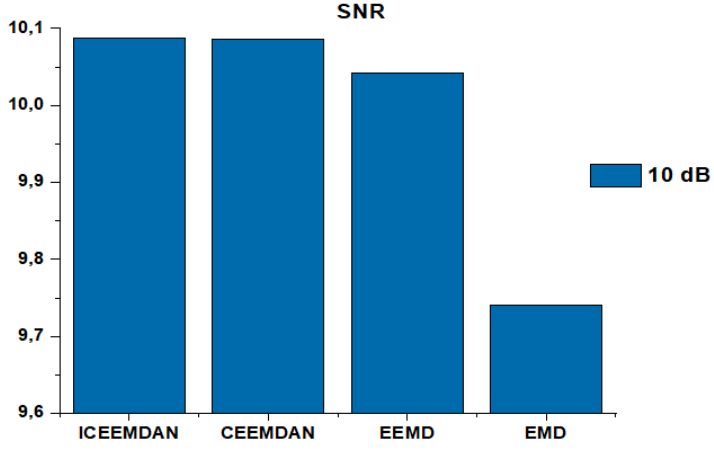

(a)

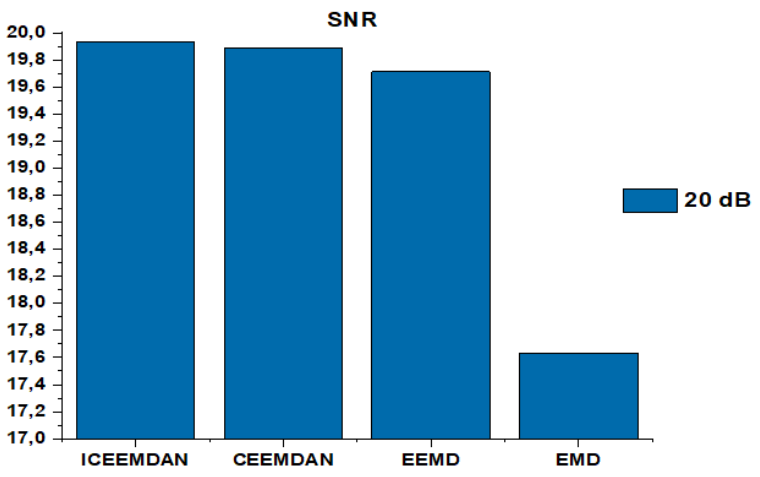

(b)

Figure 10. Diagram of SNR evaluation for normal EEG signal denoising for; (a) 10dB and (b) 20dB

\section{CONCLUSION}

The present study investigated the effect of noise on EEG and EMG signals. The proposed method is ICEEMDAN for denoising natural and artificial noise with a noise minimization property. The comparative study of EMD, EEMD, CEEMDAN, and ICEEMDAN methods is done to deduce the powerful method in terms of noise reduction used in this work using MSE and SNR metrics. According to the MSE and SNR results, the ICEEMDAN has low values of the MSE and high values of SNR. This shows its high performance compared to other methods used in this study. We conclude that the ICEEMDAN is a powerful method for reducing the noise that corrupted EEG and EMG signals.

\section{REFERENCES}

[1] L. Parrino, R. Ferri, O. Bruni, and M. G. Terzano, "Cyclic alternating pattern (CAP): The marker of sleep instability," Sleep Med Rev, vol. 16, pp. 27-45, 2012, doi: 10.1016/j.smrv.2011.02.003.

[2] L. Parrino, M. Boselli, G. P. Buccino, M. C. Spaggiari, G. Di. Giovanni, and M. G. Terzano, "The cyclic alternating pattern plays a gate-control on periodic limb movements during non-rapid eye movement sleep," J. Clin Neurophysio, vol. 13, no. 4, pp. 314-323, 1996.

[3] M. B. I. Reaz, M. S. Hussain, and Mohd-Yasin. F, "Techniques of EMG signal analysis: Detection," Biol. Proced, vol. 8, no. 1, pp. 11-35, 2006, doi: 10.1251/bpo115

[4] F. Gaetani, R. de Fazio, G. A. Zappatore, and P. Visconti, "A prosthetic limb managed by sensors-based electronic system: Experimental results on amputees," Bulletin of Electrical Engineering and Informatics, vol. 9, no. 2, pp. 514-524, April 2020, doi: 10.11591/eei.v9i2.2101

[5] A. M. NajlaIlyana, et al., "Analysis of surface electromyography for hand gesture classification," in Indonesian Journal of Electrical Engineering and Computer Science, vol. 15, no. 3, pp. 1366-1373, Sept. 2019, doi: 10.11591/ijeecs.v15.i3.pp1366-1373.

[6] S. Elouaham, R.Latif, B.Nassiri, A. Dliou, M. Laaboubi, and F. Maoulainine, "Analysis Electroencephalogram signals using ANFIS and Periodogram techniques," International Review on Computers and Software (I.RE.CO.S.), vol. 8, no. 12, pp. 2959-2966, 2013.

[7] S. Elouaham, A. Dliou, M. Laaboubi, R. Latif, N. Elkamoun, and H. Zougagh," Filtering and analyzing normal and abnormal electromyogram signals," Indonesian Journal of Electrical Engineering and Computer Science, vol. 20, no. 1, pp. 176-184, october. 2020, doi: 10.11591/ijeecs.v20.i1.pp176-184.

[8] P. Physiobank, "Physiologic signal archives for biomedical research," 2005.

[9] A. Chlögl, C. Keinrath, D. Zimmermann, R. Scherer, R. Leeb, and G. Pfurtscheller, "A fully automated correction method of EOG artifacts in EEG recordings," Clin. Neurophysiol, vol. 118, no. 1, pp. 98-104, 2007, doi: 10.1016/j.clinph.2006.09.003.

[10] A. Dliou, R. Latif, M. Laaboubi, F. M. R., Maoulainine, and S. Elouaham, "Time-frequency analysis of a noised ECG signals using empirical mode decomposition and Choi-Williams techniques," International Journal of Systems, Control and Communications, vol. 5, no. (3-4), pp. 231-245, 2013, doi: 10.1504/IJSCC.2013.058177.

[11] S. Elouaham, R. Latif, A. Dliou, F. Maoulainine, and M. Laaboubi, "Biomedical signals analysis using timefrequency," 2012 IEEE International Conference on Complex Systems (ICCS), 2012, pp. 1-6, doi: 10.1109/ICoCS.2012.6458575.

[12] T. Lee and T. Ouarda, "An EMD and PCA hybrid approach for separating noise from signal, and signal in climate change detection, " Int. J. Climatol, vol. 32, no. 4, pp. 624-34, 2012, doi: 10.1002/joc.2299

[13] A. O. Andrade, S. Nasuto, and P. Kyberd, "EMG signal filtering based on empirical mode decomposition," Biomedical Signal Processing and Control, vol. 1, no. 1, pp. 44-55, 2006, doi: 10.1016/j.bspc.2006.03.003. 
[14] C. Liu, P. X. Zhao, and J. Gao, "Reducing GPS carrier phase errors in the measurement and position domains for short-distance static relative positioning Acta Geodaet," Acta Geodaetica et Geophysica, vol. 51. pp. 81-93, 2016, doi: 10.1007/s40328-015-0111-y.

[15] D. Liu, Z. Xiao, X. Hu, C. Zhang, and O. P. Malik, "Feature extraction of rotor fault based on EEMD and curve code," Measurement, vol. 135, pp. 712-724, 2019, doi: 10.1016/j.measurement.2018.12.009.

[16] Z. Liu, Z. Y. Cui, and W. Li, "A classification method for complex power quality disturbances using EEMD and rank wavelet SVM," IEEE Trans. Smart Grid, vol. 6, no. 16, pp. 78-85, 2015, doi: 10.1109/TSG.2015.2397431

[17] A. Dliou, S. Elouaham, R. Latif, and M. Laaboub, "Combination of the CEEM Decomposition with Adaptive Noise and Periodogram Technique for ECG Signals Analysis," IntechOpen, 2019, doi: 10.5772/intechopen.86007.

[18] M. E. Torres, M. A. Colominas, G. Schlotthauer, and P. Flandrin, "A complete ensemble empirical mode decomposition with adaptive noise," in Proceedings of the 36th IEEE International Conference on Acoustics, Speech, and Signal Processing (ICASSP '11), 2011, pp. 4144-4147, doi: 10.1109/ICASSP.2011.5947265.

[19] M. Guarascio and S. Puthusserypady, "Automatic minimization of ocular artifacts from electroencephalogram: A novel approach by combining Complete EEMD with Adaptive Noise and Renyi's Entropy," Biomedical Signal Processing and Control, vol. 36, pp. 63-75, 2017, doi: 10.1016/j.bspc.2017.03.017

[20] H. Han, S. Cho, S. Kwon and S. B. Cho, "Fault Diagnosis Using Improved Complete Ensemble Empirical Mode Decomposition with Adaptive Noise and Power-Based Intrinsic Mode Function Selection Algorithm," Electronics, vol. 7, no. 2, 2018, doi: 10.3390/electronics7020016.

[21] J. Zhang, Y. Guo, Y. Shen, D. Zhaon, and M. Li, "Improved CEEMDAN-wavelet transform denoising method and its application in well logging noise reduction," Journal of Geophysics and Engineering, vol. 15, no. 3, pp. 775-787, 2018, doi: 10.1088/1742-2140/aaa076.

[22] R. A. Thuraisingham, "Revisiting ICEEMDAN and EEG rhythms," Biomedical Signal Processing and Control, vol. 68, 2021, doi: 10.1016/j.bspc.2021.102701.

[23] A. Mumtaz Ali and P. Ramendra, "Significant wave height forecasting via an extreme learning machine model integrated with improved complete ensemble empirical mode decomposition," Renewable and Sustainable Energy Reviews, vol. 104, pp. 281-295, 2019, doi: 10.1016/j.rser.2019.01.014.

[24] M. Altuve, L. Suárez, and J. Ardila, "Fundamental heart sounds analysis using improved complete ensemble EMD with adaptive noise," Biocybernetics and Biomedical Engineering, vol. 40, no. 1, pp. 426-439, 2020, doi: 10.1016/j.bbe.2019.12.007.

[25] M. Altuve, P. Lizarazo, and J. Villamizar, "Human activity recognition using improved complete ensemble EMD with adaptive noise and long short-term memory neural networks," Biocybernetics and Biomedical Engineering, vol. 40, no. 3, pp. 901-909, 2020, doi: 10.1016/j.bbe.2020.04.007. 